\title{
Subclavian vein catheterization: A rare complication
}

\author{
P. Jain, J. C. Muthusami, J. P. Raj*, P. Gaikwad, J. Rajinikanth, A. J. Tirkey
}

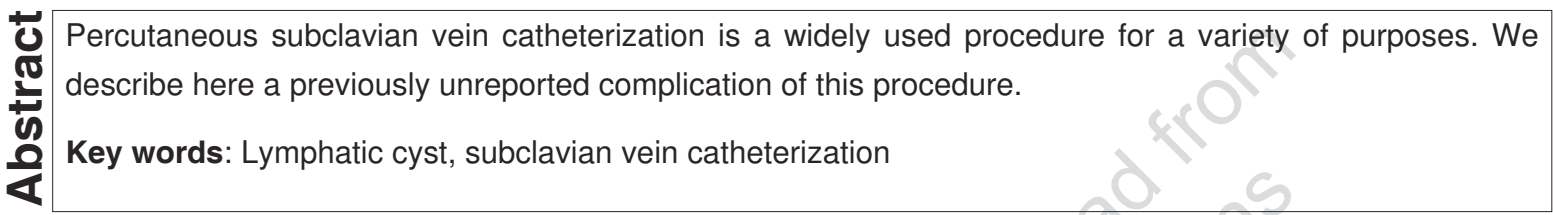

\section{Introduction}

We describe here an unusual lymphatic cyst presenting in the right infraclavicular region following subclavian vein catherization. This complication has not been previously reported. Percutaneous subclavian vein catherization is a widely used procedure for a variety of purposes. Complications have been documented extensively and include thrombosis,,$^{[1]}$ subclavian artery puncture, ${ }^{[1]}$ infection ${ }^{[2,3]}$ and perforation of the great vessels. ${ }^{[4]}$ Relatively uncommon complications include pneumothorax, ${ }^{[3]}$ pleural effusion, ${ }^{[3]}$ subcutaneous emphysema, ${ }^{[3]}$ retained fragments, ${ }^{[5]}$ chylothorax ${ }^{[6]}$ and rare complications reported include vertebral artery pseudoaneurysm. ${ }^{[7]}$

\section{Case Report}

The patient, a 21-year-old female, presented to the surgical outpatient department with a $3 \mathrm{~cm}$ spherical swelling in the right infra-clavicular region. A right subclavian vein catheter had been previously inserted percutaneously prior to a major cardiac surgery. It was placed uneventfully, functioned normally and was removed postoperatively without complication. A year later, the swelling in the right infraclavicular area appeared and grew progressively without causing any symptoms. It was aspirated at a general hospital and disappeared. A

\section{From:}

General Surgery Unit 1 and *Surgical ICU, CMCH Vellore, India

\section{Correspondence:}

Dr. John Muthuisami, Prof \& Head, Department of General Surgery Unit 1, CMC, Vellore, India. E-mail: sur1@cmcvellore.ac.in year later it recurred without causing any other symptoms. The Magnetic Resonance Imaging scans done at this hospital revealed a cystic swelling that was encasing the right subclavian and adjoining axillary vessels [Figure 1]. She underwent an excision of the swelling under general anesthesia, preserving the vessels. The cyst contained straw colored fluid in keeping with its lymphatic origin. On histopathological examination, the cyst was found to have a wall consisting of fibrous elements and occasional angiomatous components. The postoperative period was uneventful and the patient was discharged on the third postoperative day.

\section{Discussion}

We highlight here a previously unreported complication of subclavian vein catherization.

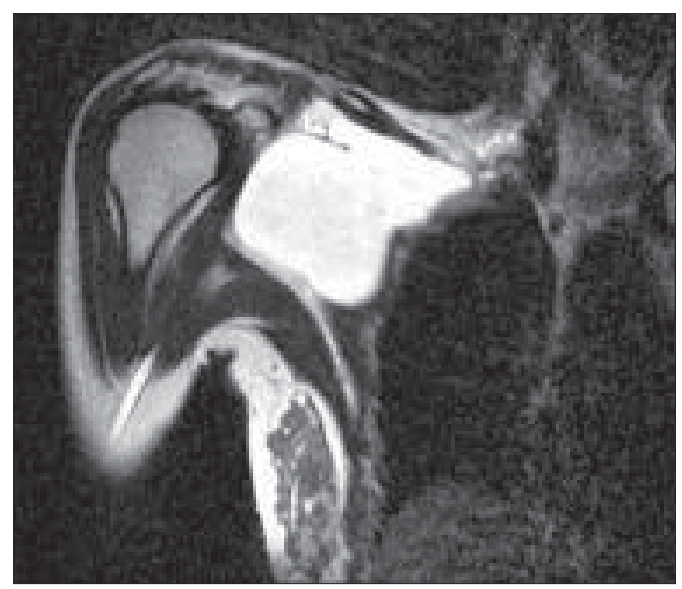

Figure 1: Magnetic resonance T2 contrast image showing cyst in the right infraclavicular area 
It is probable that when the subclavian vein catheter was placed, it could have damaged the right lymph trunk at the point where it joined the subclavian vein. The damage would have been minor enough to not cause immediate symptoms, but large enough to cause lymphatic fluid to accumulate slowly. The space being sufficient for a significant swelling to arise and the progress slow allowing fibrous elements to encase the fluid.

The recent onset and medical history pointed towards the subclavian catheterization as a possible cause of the problem. Clinical examination showed a cystic swelling deep to the pectoralis major. The Magnetic Resonance Imaging scans confirmed the position of the swelling and encasement of the vessels. At surgery, the presence of straw-colored fluid corroborated the idea of this being a lymph collection. Histopathology confirmed the diagnosis of an acquired lymphatic cyst.

This is a rare occurrence and we recommend that lymphatic cyst formation be added to the list of complications relating to subclavian vein catherization.

\section{References}

1. Mitchell SE, Clark RA. Complications of central venous catheterization. Am J Roentgenol 1979;133:467-76.

2. Purdue GF, Hunt JL. Placements and complications of monitoring catheters. Surg Clin North Am 1991;71:723-31.

3. Herbst CA Jr. Indications, management and complications of percutaneous subclavian catheters. Arch Surg 1978;113:14215.

4. Robinson JF, Robinson WA, Cohn A, Garg K, Armstrong JD $2^{\text {nd }}$. Perforation of the great vessels during central venous line placement. Arch Intern Med 1995;155:1225-8.

5. Jones SA, Giacomantonio M. A complication associated with central line removal in the pediatric population: Retained fixed catheter fragments. J Pediatr Surg 2003;38:594-6.

6. Van Veldhuizen PJ, Taylor S. Chylothorax: A complication of a left subclavian vein thrombosis. Am J Clin Oncol 1996;19:99-101.

7. Amaral JF, Grigoriev VE, Dorfman GS, Carney WI Jr. Vertebral artery pseudoaneursym. A rare complication of subclavian artery catheterization. Arch Surg 1990;125:546-7.

Source of Support: Nil, Conflict of Interest: None declared. 